\title{
Las concesiones y la optimización del transporte vial y ferroviario
}

\section{lan Thomson}

Unidad de Transporte, División de Comercio

Internacional, Transporte

y Financiamiento, CEPAL
Los ferrocarriles latinoamericanos enfrentaron por primera vez una competencia importante de otro medio terrestre en el decenio de 1930, uno de cuyos resultados fue una pérdida significativa de los ingresos generados por el transporte de productos de valores unitarios relativamente altos. Estos ingresos solventaban sus gastos fijos de administración e infraestructura. Las dificultades financieras de las empresas las empujaron hacia el sector estatal. En los siguientes decenios fueron perdiendo paulatinamente su encanto para los gobiernos a raíz de sus déficit financieros y su papel decreciente en la economía nacional. Se continuó eliminando progresivamente los servicios de carga de menor volumen y los de pasajeros, que ya habían llegado a ser bastante escasos cuando los gobiernos decidieron devolver los ferrocarriles al sector privado a partir de fines de los años setenta. La creciente dedicación de los ferrocarriles al transporte de carga voluminosa entre un número reducido de puntos específicos hace que, en corredores sin vías navegables, el competidor casi único sea hoy el camión pesado. Las subvenciones al tránsito de camiones pesados han sido reducidas, aunque no dificultadas, por las concesiones de carreteras. Dentro de pocos años será factible, en el sentido tecnológico, cobrar a los camiones pesados peajes que reflejen adecuadamente los costos externos de su circulación, como los del desgaste infraestructural. Entretanto, podría crearse una igualdad de condiciones competitivas entre los medios ferroviario y carretero mediante subsidios compensatorios al primero. Esto dirigiría cada tráfico hacia el medio que lo pueda transportar con menor costo. Sin embargo, para que los beneficios sean percibidos por la comunidad en general, más que por las empresas concesionarias ferroviarias o de carreteras que forman parte del sector privado, es aconsejable establecer una política de compensaciones antes de licitar las concesiones. 


\section{I}

\section{Antecedentes históricos}

\section{La tarificación ferroviaria antes del surgimien- to de la competencia caminera}

En lo que se refiere al transporte terrestre, hasta la aparición de los ferrocarriles los medios camineros no enfrentaron competencia alguna. En algunos países, incluidos muchos de Europa, los canales y ríos se presentaron como opciones viables, y en las Américas, en las épocas precolombina y colonial cuando, especialmente en la era de dominio ibérico, una gran parte del desarrollo socioeconómico ocurría en las fajas litorales y en las zonas donde se había encontrado alguna riqueza mineral, el cabotaje frecuentemente fue preferido a los primitivos caminos.

Con la llegada al escenario de los ferrocarriles hubo un salto tecnológico de bastante importancia; antes de la inauguración de la locomotora a vapor, el ser humano no había producido una máquina que se pudiera automovilizar. La venida de los ferrocarriles trajo consigo una reducción muy significativa de los costos de transporte, y efectivamente, salvo para movimientos de muy corta distancia, en cada corredor donde había ferrocarril casi dejó de existir el transporte caminero. La posición monopólica de los ferrocarriles atrajo la atención de los incipientes ministerios de economía, que empezaron a aplicarles reglamentos que restringieron su libertad de acción en materia tarifaria (Thomson, 1998). Aun en las instancias en que las empresas ferroviarias siguieron gozando de alguna flexibilidad con relación a sus tarifas, tendieron a cobrar fletes relativamente altos al transporte de productos cuyo valor unitario era también comparativamente alto, lo que tuvo una cierta lógica comercial, siempre que no enfrentaran los ferrocarriles una competencia significativa. En 1886 un observador comentó que "Todos los ferrocarriles del mundo han aceptado las tarifas diferenciadas, porque de otro modo las mercaderías de poco valor no podrían soportar los subidos fletes de largas distancias" (EFE, 1887). En 1932 la empresa Ferrocarriles del Estado de Chile, cobraba 36 centavos la tonelada/kilómetro por el transporte de aguardientes, alcoholes y licores, 21 centavos por el de vinos, y solamente 15 centavos por el de aguas minerales, aunque el costo de transportar cualquiera de esas categorías de bebidas habría sido muy parecido al de las demás.
Mediante el transporte de productos de mayor valor unitario, las empresas ferroviarias pudieron financiar no sólo los costos directos del transporte propiamente tal, sino también los gastos fijos de infraestructura, administración, etc. Cabe aclarar que la proporción del valor de venta del producto que representaba el flete era aún menor en el caso de los productos de mayor valor en el mercado, aunque el valor absoluto de ese flete era muy superior al pagado por los productos de menor precio.

\section{El impacto sobre los fletes ferroviarios de la competencia, y sus consecuencias}

Los problemas económicos de las empresas ferroviarias comenzaron, en general, con el surgimiento de la competencia caminera, que se interesó especialmente en el mercado de los mismos productos cuyo traslado permitía a las empresas ferroviarias cubrir sus costos fijos, además de modernizarse y pagar dividendos de valores que permitiesen mantener el interés de sus accionistas. El momento en que ocurrió este fenómeno no fue el mismo para todos los ferrocarriles; entre Santiago y Valparaíso, donde el volumen de carga transportada por los camiones casi se triplicó entre 1934 y 1937, se inició hace mucho tiempo (Huidobro, 1939); por otra parte, todavía no ha ocurrido en todos los casos, como en las rutas ferroviarias internacionales entre Salta y Baquedano, y de São Paulo a Santa Cruz de la Sierra, donde aún no existen buenas carreteras. En el cuadro 1 se ilustra el fenómeno en el caso de la sección chilena del Ferrocarril de Arica a La Paz. Entre los años a los cuales se refiere el cuadro, es decir, entre 1992 y 1996, se completó la pavimentación de la carretera que vincula esas dos ciudades, lo que aumentó fuertemente la competencia caminera. Los fletes ferroviarios de todos los productos incluidos en el cuadro bajaron en términos reales, pero los que bajaron más fueron los que en 1992 eran más altos, para los productos de valores unitarios también relativamente altos, como los vehículos motorizados y los productos manufacturados en contenedores.

Este fenómeno explica en gran parte que en un gran número de países los ferrocarriles se incorporaran al sector gubernamental entre 1935 y 1965. En ese período, llegó a ser evidente que las empresas priva- 
CUADRO 1

Ferrocarril de Arica a La Paz, sección chilena: Relación entre el valor absoluto del flete y cambio relativo del mismo, 1992-1996

\begin{tabular}{lcc}
\hline Producto & $\begin{array}{c}\text { Flete sobre el } \\
\text { producto como \% del } \\
\text { flete promedio, 1992 }\end{array}$ & $\begin{array}{c}\text { Cambio en el } \\
\text { valor real del flete, } \\
1992 \text { a 1996 (\%) }\end{array}$ \\
\hline Trigo & 91 & -33 \\
Petróleo diesel & 122 & -45 \\
Harina de trigo & 85 & -20 \\
Vehículos & 270 & -44 \\
Productos & & \\
manufacturados & 120 & -58 \\
en contenedores & 58 & -21 \\
Zinc & 60 & -21 \\
Plomo & & -27 \\
Papel y productos & 95 & \\
de celulosa & & \\
\hline
\end{tabular}

Fuente: Elaboración propia, sobre la base de datos presentados en EFE (varios años).

das no podían atraer capital para competir en el largo plazo, y a veces ni siquiera podían solventar sus operaciones en el corto plazo, al menos mientras siguiesen manteniendo servicios de altos costos unitarios, como los de ramales. En algunas ocasiones - por ejemplo en Paraguay - el ferrocarril tuvo que dejar de operar antes de que el gobierno se diera cuenta de que tenía un valor socioeconómico o estratégico que superaba su déficit financiero. Sin el ferrocarril, los grandes volúmenes de productos de bajo valor, y por ende de bajos fletes, enfrentarían serias dificultades para encontrar una manera de movilizarse a los puertos, a las fábricas o a las zonas de consumo. De la misma manera, se complicarían bastante los desplazamientos de los ciudadanos de menores ingresos.

En manos de los gobiernos, los ferrocarriles a menudo sufrieron las consecuencias adversas de insuficiencias en su administración y gestión, y en algunos casos, como en Argentina, fueron sometidos a una intervención motivada políticamente que comprometió con fuerza su eficiencia económica. Los gerentes no recibieron de los gobiernos instrucciones claras respecto a la importancia relativa que debía asignarse al ofrecimiento de un servicio social o de fomento económico, y a la cobertura de los costos con los ingresos, lo que tendió a reducir aún más la eficiencia de la gestión. Sin embargo, por no tener obligación de autofinanciarse, casi siempre lograron sobrevivir y, en casos importantes, mediante la inyección de fondos provenientes de los gobiernos o de préstamos garantizados por éstos, modernizarse en el sentido tecnológi- co, pero no en el institucional. Se tendió a enfocar la modernización hacia aquellas áreas que más llamaran la atención de los votantes, como los coches y las locomotoras, relegándose a un plano inferior la inversión en las vías.

Por no tener ya que solventar todos sus gastos y, además, por el deseo de los gobiernos de fomentar el desarrollo de distintos sectores geográficos o económicos, muchos fletes cobrados tendieron a reflejar meramente los costos marginales relacionados con el transporte (y, según cuentan algunos gerentes de ferrocarriles recientemente privatizados, a veces ni cubrieron los costos marginales).

\section{Los gobiernos devuelven los ferrocarriles al sector privado}

Al llegar a mediados de los años ochenta, muchos gobiernos, como los de Argentina o Brasil, habían empezado a disminuir fuertemente sus inversiones en los ferrocarriles y, a fines de la misma década, comenzaron a preguntarse por qué estaban canalizando cuantiosos recursos a equilibrar las cuentas de entidades ferroviarias cuyo papel en la economía nacional parecía reducirse año tras año. En América Latina, el primer gobierno en hacerse esta pregunta fue el colombiano, luego el argentino y después la mayoría de los demás, lo que desembocó en la privatización de los ferrocarriles, normalmente por la vía de concesiones. A veces, tal como había ocurrido treinta años antes en Paraguay, por el paso de la administración privada a la estatal, o por su devolución a manos privadas, también el ferrocarril dejó de operar durante un período, como sucedió en Guatemala y Costa Rica.

\section{Las actitudes comerciales de las empresas privadas}

Los nuevos gerentes ferroviarios del sector privado no tuvieron demasiado interés en transportar carga por fletes iguales o inferiores a los costos marginales, y esperaban que todos los tráficos atendidos contribuyesen a lo menos algo a la financiación de los costos fijos. Por lo tanto, aunque con la privatización los volúmenes de transporte, en general, han tendido a subir, también algunas cargas han dejado de transportarse por ferrocarril. Un caso ilustrativo es el de la empresa chilena Ferronor, que ha negociado un contrato para el transporte a granel de cinco millones de toneladas de mineral de fierro entre la mina de Los Colorados y el puerto de Huasco, mientras ha dejado de atender 
tráficos de poco volumen y aun menos rentabilidad, como el de cemento de La Calera a Copiapó, o de automóviles desde Iquique a Barranqueros, en la Argentina.

Al dejar de transportarse por ferrocarril, el tráfico opta por otro medio de transporte, o sencillamente deja de existir, trayendo, en cualquiera de estas dos instancias, consecuencias desafortunadas. Si se desplaza a otro medio, los costos económicos podrían ser superiores a los de su transporte por ferrocarril, y si deja de existir, será probable que haya recursos ociosos, a lo menos en el corto plazo.

Es importante destacar que la creciente concentración de las empresas ferroviarias en los tráficos más rentables, que en general son los más indicados para transportarse por ferrocarril, significa que los buses interurbanos y los camiones de menor tonelaje ya no compiten con los trenes. Cada vez más, las empresas que compiten con las empresas ferroviarias son las empresas o los individuos que operan los camiones de mayor tonelaje.

\section{Un caso concreto de materialización de los beneficios socioeconómicos del ferrocarril}

Usando como ejemplo otra vez el caso de Ferronor, en 1986 el gobierno militar de la época, cansado de los déficit de ese ferrocarril, autorizó por decreto el levantamiento de casi la totalidad de los $850 \mathrm{~km}$ más sureños de su línea principal (Thomson, 1997). Se determinó el cierre, más bien que la privatización, porque ésta aún no había llegado a considerarse como opción viable en Chile, y tampoco en el resto del mundo. El poco tráfico que corría en ese período sobre el tramo cuyo levantamiento se aprobó, entre La Calera y Copiapó, consistía casi exclusivamente de minerales de cobre, a raíz de un desequilibrio en la distribución geográfica de la capacidad de concentración de los minerales, por una parte, y de refinamiento, por la otra.

En ese momento, en la CEPAL se elaboraba una metodología que permitiera comparar los costos del desgaste de las carreteras causado por los vehículos pesados; los pagos que efectuaban éstos por concepto de peajes, si los hubiera, y los impuestos, como el que gravaba los combustibles (CEPAL, 1987a). Se aplicó la metodología al transporte por camión de la carga llevada en esos momentos sobre el tramo de Ferronor cuyo levantamiento se había autorizado, determinándose que cada tonelada-km transportada por los camiones representaba un costo neto para el gobierno equivalente a unos 0.41 centavos de dólar (222 000 dólares al mes) a precios corrientes. ${ }^{1}$ Es decir, si el subsidio necesario para mantener en operación el ferrocarril fuera inferior a este monto, desde el punto de vista de la comunidad convendría pagarlo con el fin de evitar un mayor desgaste a las carreteras. Pocos meses después, el gobierno de la época decidió emitir un segundo decreto, dejando sin efecto el anterior.

\section{II}

\section{Los peajes camineros y los costos}

\section{de la infraestructura vial}

\section{El desgaste de las carreteras por el tránsito pesado}

Es generalmente reconocido que el tránsito de los vehículos pesados causa daños estructurales a las carreteras, los que varían exponencialmente de acuerdo con el peso por eje de los vehículos. El valor del exponente varía según las circunstancias entre 2.4 y 6.6; se considera que normalmente el valor se aproxima a 4.5 (Rolt, 1981). El valor de respaldo (default) en el modelo para el diseño y mantenimiento de carreteras del Banco Mundial (modelo HDM) es de 4.0. En éste se identifica un eje equivalente (EE) como el poder destructivo de un eje cargado con un peso de 18000 libras (equivalentes a 8165 toneladas), siendo éste la unidad básica de análisis. Un camión de dos ejes, cargados con 5.0 y 6.0 toneladas, respectivamente, suponiendo que el valor del exponente es de 4.5, tiene un valor en ejes equivalentes de 0.36 . El mismo camión cargado con 7.5 toneladas sobre el eje delantero y 15.0

\footnotetext{
${ }^{1}$ En 1992 Ferronor tuvo un resultado operacional favorable, y en 1993 su déficit fue de 272000 dólares al mes.
} 
sobre el trasero, tiene una equivalencia de 16.1 , y si se cargan 2.5 toneladas adicionales al eje trasero, la equivalencia sube a 31.6. La equivalencia en ejes fluctúa un poco según la calidad del pavimento, y, en general, un camión con un valor determinado en ejes equivalentes, causa más daño en una vía de calidad inferior que en otra de mejor calidad.

\section{La estructura de los peajes en las carreteras latinoamericanas}

En algunos países de América Latina, como Perú y Chile, desde hace varios años, o decenios, las autoridades públicas han cobrado peajes por el uso de algunos tramos de la red vial, especialmente las carreteras principales con estándares de construcción relativamente altos. Más recientemente, el cobro de peajes se está convirtiendo en un fenómeno más común, al entregarse en concesión la construcción o reconstrucción, el mantenimiento y la administración de las carreteras a empresas del sector privado.

Como hemos visto, una mera modificación en la estiba, que coloque un poco menos peso sobre el eje menos cargado de un camión de dos ejes, desplazándolo al otro eje sin cambiar el peso bruto total del vehículo, puede incrementar significativamente el valor en ejes equivalentes. Para condiciones de estiba que se podrían describir como ideales, el cuadro 2 señala el valor en ejes equivalentes de tres tipos distintos de camiones, cargados con dos niveles alternativos de peso, y que circulan sobre, en un caso, una carretera de asfalto y, en el otro, una de hormigón. Aun en condiciones de estiba ideal, es evidente que el desgaste ocasionado a una carretera depende de manera crítica del peso por eje de los vehículos que la ocupan.

Cabe observar, por ejemplo, que el valor en ejes equivalentes de un camión con peso bruto de 35 toneladas y cuatro ejes es de 2.39 (asfalto) ó 3.02 (hormigón), mientras un camión del mismo tonelaje y de cinco ejes tiene una equivalencia de 1.74 ó de 2.02, respectivamente.

La estructura de los peajes por tipo de vehículos presenta relativamente poca variación entre los diferentes países. Por lo general, al licitar la concesión de una carretera, el gobierno fija la relación entre el peaje cobrado a un vehículo de tipo $i$ (por ejemplo, camión liviano, camión rígido de tres ejes, camión semirremolque de tres ejes, bus de dos ejes) y el que se aplica a un automóvil, y usa el peaje por automóvil como la variable crítica en la adjudicación de la concesión: ésta se adjudica al interesado que ofrezca cobrar el menor peaje por automóvil.

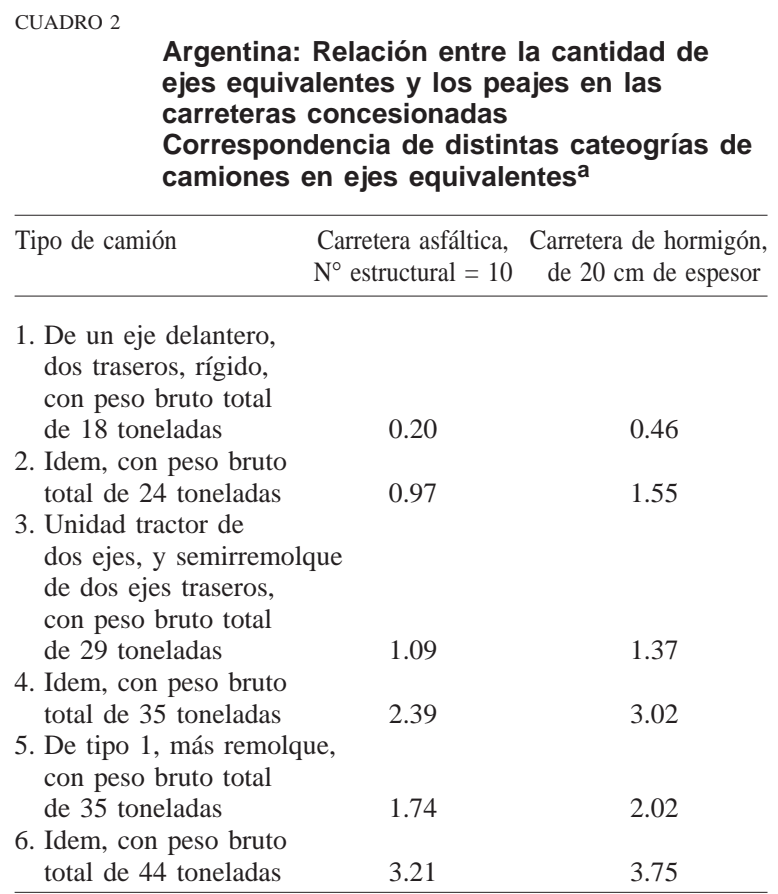

Fuentes: Chile, Comisión Nacional de Energía (1981) a través de CEPAL, 1987a.

a Suponiendo una estiba ideal, es decir, con una distribución de peso por eje según el máximo permitido por la ley.

\section{La relación entre los peajes y los costos mar- ginales de uso de la infraestructura}

Es evidente que la variación en el valor del peaje entre los diferentes tipos de vehículos no refleja la variación correspondiente de los valores en ejes equivalentes. En el cuadro 3 se presenta la estructura de los peajes de las carreteras concesionadas en la Argentina y en el cuadro 4 la de Brasil; en términos generales, la situación se repite en el caso de las vías con peaje de Colombia, Chile o México.

En el caso de las vías sin peaje, salvo la adquisión de combustible, servicios de mantenimiento y otros recursos económico requeridos para cualquier viaje en vehículo, virtualmente el único pago adicional es el impuesto sobre el combustible, cuyo valor frecuentemente muestra, en algún sentido, una relación inversa con el desgaste causado a la infraestructura vial, ya que grava más la gasolina que el diesel.

Se concluye, aun en los casos en que se cobran peajes, que a los automóviles y otros vehículos livianos se les cobra excesivamente en relación con el valor del daño que ocasionan a las carreteras. Por otra 
CUADRO

Argentina: Relación entre la cantidad de ejes equivalentes y los peajes en las carreteras concesionadas

\begin{tabular}{ccc}
\hline Tipo de vehículo & Cantidad & Peaje máximo \\
indicativa de & en horario \\
ejes equivalentes & normal \% \\
\hline
\end{tabular}

De hasta dos ejes y 2.10 metros

de altura, sin rueda doble

De hasta dos ejes y de más de

2.10 metros de altura o rueda doble

De más de dos y hasta cuatro ejes,

de menos de 2.10 metros de altura

o rueda doble

De más de dos y hasta cuatro ejes,

de más de 2.10 metros de altura o

rueda doble

De más de cuatro y hasta seis ejes

o rueda doble

Vehículos de más de seis ejes y de

más de 2.10 metros de altura o

rueda doble

$\begin{array}{cc}- & 100 \\ 1.50 & 200 \\ 3.00 & 200 \\ 4.00 & 300 \\ 4.00 & 400 \\ 4.00 & 500\end{array}$

Fuentes: Elaboración propia, en el caso de los ejes equivalentes, usando como referencias: Archondo (1989), Banco Mundial (1989, cuadro 6-11), CEPAL (1987 a y b) y Chile, Comisón Nacional de Energía (1981): los valores de los peajes relativos provienen de FLACSO, 1998.

CUADRO 4

Brasil: Relación entre la cantidad de ejes equivalentes y los peajes en las carreteras concesionadas ${ }^{a}$

\begin{tabular}{ccc}
\hline Tipo de vehículo & $\begin{array}{c}\text { Cantidad } \\
\text { indicativa de ejes } \\
\text { equivalentes }\end{array}$ & $\begin{array}{c}\text { Peaje máximo } \\
\text { en horario normal } \\
(\text { auto }=100 \%)\end{array}$
\end{tabular}

Automóvil, camioneta o furgón

100

Camión o bus de dos ejes y

neumáticos traseros duplicados

$-$

Automóvil o camioneta con

1.87

200

semirremolque, de tres ejes y

neumáticos traseros sencillos

Bus o camión articulado, de

tres ejes y neumáticos traseros

duplicados

300

Automóvil o camioneta con

remolque, cuatro ejes, neumáticos

sencillos

Camión articulado de cuatro ejes

y neumáticos duplicados

Idem, de cinco ejes

Idem, de seis ejes

Moto o motoneta

Fuentes: Elaboración propia, en el caso de los ejes equivalentes, usando como referencias: Archondo (1989), Banco Mundial (1989), cuadro 6-11), CEPAL (1987 a y b) y Chile, Comisión Nacional de Energía (1981): los valores de los peajes relativos provienen del trabajo de Pereira (1997).

a Se eximen de pago los vehículos oficiales y, además, los pertenecientes a las fuerzas armadas y la policía militar. parte, los cobros a los camiones más pesados, por eje, son muy modestos en comparación con el costo del daño que estos vehículos causan.

\section{4. ¿El tránsito de los camiones pesados está subvencionado?}

La conclusión del párrafo anterior no significa necesariamente que exista una subvención implícita al tránsito de los camiones pesados, ya que es posible que a todos los vehículos se les cobren montos superiores a los costos correspondientes. Es decir, se admite la posibilidad de que exista un gravamen positivo (o sea, un subsidio negativo) al tránsito de todos los tipos de vehículos, siendo mayor ese gravamen para los automóviles que para vehículos más pesados. Sin embargo, esta no parece ser la situación.

En el cuadro 5, que se refiere a las carreteras concesionadas en Chile, la comparación de los valores de las últimas dos columnas muestra que sólo en el caso de los camiones, y especialmente de los de más de dos ejes, el costo del desgaste supera la suma del impuesto específico sobre el combustible y el peaje. ${ }^{2}$

Cabe recordar que en Chile los impuestos sobre el diesel son más altos que en algunos otros países latinoamericanos. Evidentemente, en países donde dichos impuestos sean menores que en Chile, habrá más probabilidades de que el tránsito de los distintos tipos de vehículos cause un costo de desgaste de las vías que supere su contribución a través de los peajes e impuestos que pagan.

En el caso de los camiones y también de los buses de dos ejes, desde el punto de vista del concesionario, el costo del desgaste de la carretera es mayor que su recaudación por peajes. Y, evidentemente, en el caso de las carreteras sin peaje, la circulación de los camiones y buses tanto de dos ejes como de más de dos ejes origina un costo por desgaste de la vía que sobrepasa los montos contribuidos a través del impuesto específico sobre los combustibles.

\footnotetext{
${ }^{2}$ El cuadro 5 se debería interpretar como una ilustración conceptual más que como una reflexión fiel de la situación en un caso específico. Es probable, por ejemplo, que los valores en ejes equivalentes de los vehículos que transitan sobre las carreteras concesionadas no sean exactamente iguales que los considerados en los cálculos resumidos en el cuadro. Además, cabe aclarar que en él no se toman en cuenta el impuesto al valor agregado ni los costos variables de policía y otros.
} 
CUADRO 5

Chile: Relación entre la cantidad de ejes equivalentes

y los peajes en las carreteras concesionadas

\begin{tabular}{|c|c|c|c|c|c|}
\hline Tipo de vehículo & $\begin{array}{c}\text { Cantidad } \\
\text { indicativa de ejes } \\
\text { equivalentes }\end{array}$ & $\begin{array}{c}\text { Peaje } \\
\text { (dólares } / \mathrm{km} \text { ) }\end{array}$ & $\begin{array}{l}\text { Impuesto sobre } \\
\text { el combustible } \\
\text { (dólares/km) }\end{array}$ & $\begin{array}{c}\text { Suma de las dos } \\
\text { columnas anteriores } \\
\text { (dólares } / \mathrm{km})\end{array}$ & $\begin{array}{c}\text { Costo de degaste } \\
\text { de la carretera } \\
\text { (dólares } / \mathrm{km} \text { ) }\end{array}$ \\
\hline Autos & - & 0.0231 & 0.0176 & 0.0407 & - \\
\hline Camionetas & 0.10 & 0.0231 & 0.0220 & 0.0451 & 0.0046 \\
\hline Camiones de dos ejes & 1.87 & 0.0416 & 0.0286 & 0.0702 & 0.0851 \\
\hline Camiones de más de dos ejes & 4.00 & 0.0925 & 0.0400 & 0.1325 & 0.1820 \\
\hline Buses de dos ejes & 1.25 & 0.0416 & 0.0333 & 0.0749 & 0.0569 \\
\hline Buses de más de dos ejes & 1.00 & 0.0717 & 0.0400 & 0.1117 & 0.0455 \\
\hline Autos y camionetas con remolque & 0.10 & 0.0347 & 0.0275 & 0.0622 & 0.0046 \\
\hline
\end{tabular}

Fuente: Elaboración propia, sobre la base de los cuadros anteriores y sus respectivas fuentes; Transporte Moderno, 1996; FMI (varios años), e información de Alberto Bull, consultor de la CEPAL.

\section{Las concesiones subvencionadas}

Otro factor que ha de tomarse en cuenta al decidir si hay o no subvención al transporte camionero, es si existe o no una subvención generalizada al concesionario. Sobre este punto es muy difícil llegar a conclusiones definitivas. En algunos casos — por ejemplo, en Chile en los tramos Santiago-Talca y Talca-Chillán de la carretera longitudinal sur-, el concesionario está obligado a efectuar un pago positivo al Estado; en otros tramos con menor tránsito y/o mayores costos de inversión, es el Estado el que paga al concesionario (cuadro 6).

En Colombia ha habido subsidios a carreteras concesionadas. En la Argentina, el gobierno no cobra canon y diversas concesiones disfrutan de subvencio-

CUADRO 6

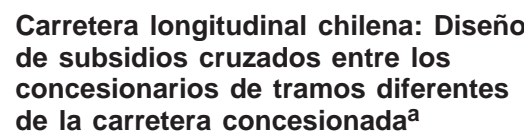

\begin{tabular}{ll}
\hline Tramo & Subsidio (-) o pago $(+)$
\end{tabular}
en dólares $\div 10^{6}$

\begin{tabular}{lr} 
La Serena-Los Vilos & -147 \\
Los Vilos-Santiago & -20 \\
Santiago-Talca/Autopista & \\
Santiago-San Fernando & +228 \\
Talca-Chillán & +166 \\
Chillán-Collipulli & -95 \\
Collipulli-Temuco & +91 \\
Temuco-Río Bueno & -94 \\
Río Bueno-Puerto Montt & -129 \\
\hline
\end{tabular}

Fuente: Friedmann e Hinojosa, sin fecha.

a Ha habido algunas diferencias entre los valores contemplados y los realmente contratados. nes, aunque hay atraso en su pago. ${ }^{3}$ En México se han pagado fuertes subsidios a los concesionarios. En el túnel El Melón, en Chile, el concesionario debe efectuar un pago al gobierno. Es evidente que la situación varía mucho de un caso a otro. Sin embargo, aunque hay pocas referencias específicas a la materia, parece probable que cualquier subsidio que se pague tenga como propósito el de financiar la inversión inicial, una ampliación de capacidad o la mejora de los estándares de diseño, y no el de mantener la carretera una vez realizada esta inversión inicial.

\section{Algunas conclusiones preliminares}

De lo anterior, se puede concluir lo siguiente:

i) el tránsito camionero sobre vías sin peaje es casi siempre subvencionado, en el sentido de que los montos contribuidos mediante los impuestos específicos sobre los combustibles, y los demás que varían en el margen con el kilometraje recorrido, son inferiores que el costo del desgaste correspondiente ocasionado a las vías;

ii) en el caso de las carreteras concesionadas, es probable que existan subvenciones implícitas a los camiones de mayores pesos por eje, ${ }^{4} \mathrm{y}$,

\footnotetext{
${ }^{3}$ Una fuente gremial autobusera considera que el subsidio moroso en 1998 asciende a unos 45 millones de dólares. Véase Cámara Empresarial de Larga Distancia, 1998.

${ }^{4}$ La fuente de la subvención depende del caso. Si se concesiona una carretera sin subvención o canon, y el costo del desgsate atribuible a los camiones supera el monto que ellos contribuyen por concepto de peajes, es evidente la existencia de una subvención cruzada, financiada por los motoristas y los ocupantes de otros vehículos. Si la concesión es subsidiada, es posible que la fuente sea la comunidad en general.
} 
iii) la materia es compleja, y las variaciones entre un caso y otro podrían ser significativas, lo que justifica su análisis también caso por caso.

\section{La significación de las subvenciones a los camiones pesados en las finanzas ferroviarias}

Una subvención implícita del orden de 0.05 dólares por km a un camión de más de dos ejes que circula sobre una vía concesionada con peaje, corresponde aproximadamente a una de 0.25 centavos $\mathrm{t} / \mathrm{km}$ neta. Cabe preguntarse si este valor es cuantitativamente significativo.

Usemos como caso ilustrativo el del Ferrocarril del Pacífico chileno (FdP), que transporta anualmente alrededor de 800 millones de t/km netos; si pudiera ingresar unos 2 millones de dólares adicionales al año, es decir, si recibiera una compensación del mismo valor de 0.25 dólares por $\mathrm{t} / \mathrm{km}$ transportada, sus ingresos subirían en un 6\%. Actualmente, el FdP tiene delicadamente equilibrados sus costos e ingresos y un aumento en estos últimos en torno a un $6 \%$ fortalecería la condición económica de la empresa en el largo plazo.

También se puede citar como ejemplo el caso de Ferronor en el año 1995 (mientras todavía estaba en manos estatales). ${ }^{5} \mathrm{Si}$ hubiera podido recibir 0.25 dólares adicionales por cada $\mathrm{t} / \mathrm{km}$ transportada, en total sus ingresos habrían subido unos 750000 dólares, y la rentabilidad del valor del activo neto se habría elevado de 0.2 a $1.7 \%$. En el caso particular de Ferronor, las carreteras de la zona geográfica en que éste opera no están sujetas a peajes, de modo que la subvención efectiva a la competencia camionera era bastante más que 0.25 dólares por cada $\mathrm{t} / \mathrm{km}$, y probablemente haya sido de alrededor de 0.71 dólares por cada $\mathrm{t} / \mathrm{km}$. Si Ferronor hubiera podido elevar sus fletes en este último valor, su rentabilidad habría subido a $4.6 \%$.

Es decir, aunque sea proporcionalmente reducido el impacto sobre las finanzas ferroviarias de los subsidios efectivamente otorgados al tránsito de los camiones pesados, sobre todo cuando se pagan peajes y aunque se concesionen las carreteras, puede influir de manera significativa en la situación económica de las empresas ferroviarias. Además, al mejorar la situación

\footnotetext{
${ }^{5}$ Ferronor está en manos privadas desde principios de 1997. Usamos el año 1995 para estimar el impacto de la subvención al transporte camionero sobre las finanzas de la empresa, a raíz de la mayor disponibilidad de datos estadísticos durante su etapa de empresa pública.
}

financiera de los ferrocarriles, debería permitir que éstos compitieran mejor en el mercado y absorbieran una mayor proporción del volumen de carga transportado.

\section{La factibilidad de cobrar peajes justos a los camiones pesados}

En la práctica, la estructura de los peajes en las carreteras latinoamericanas tiene más que ver con la facilitación del pago que con el cobro de valores que reflejen los costos del desgaste ocasionado a las vías. Los empleados que cobran los peajes estarían perfectamente capacitados para verificar el número de ejes de un camión que pasa por la plaza donde trabaja, pero normalmente no estarían en condiciones de estimar el valor en ejes equivalentes del camión. Por lo tanto, podrían aplicar una estructura de peajes que use como variable independiente el número de ejes de un camión, pero no una que haga depender el valor cobrado de la equivalencia en ejes de cada vehículo.

En algunos países, como Islandia y Nueva Zelandia, ya se venden licencias para circular sobre la red vial por mil km o múltiplos de mil km, cuyo valor depende del valor en ejes equivalentes del camión (CEPAL, 1993). Sin embargo, por razones culturales, este tipo de sistema podría no funcionar eficientemente en América Latina.

Por otra parte, no es técnicamente imposible dotar a cada plaza de peaje de una romana que pueda pesar cada eje de los vehículos que circulan sobre ella a baja velocidad. Este tipo de instalaciones ya se ocupa en las carreteras principales de muchos países, con el fin de fiscalizar las normas sobre pesos máximos autorizados. Asimismo, la tecnología actual permitiría tanto pesar cada eje como cobrar automáticamente el valor correspondiente, restando una fracción de un monto depositado con anterioridad, mediante una tarjeta inteligente insertada en un dispositivo que se coloca en la parte superior del parabrisas del camión (CEPAL, 1999). Es decir, ya puede pensarse que en un futuro relativamente cercano se podrá aplicar estructuras de peajes que cobren a cada vehículo un monto que refleje el costo del daño que haya causado a la carretera que ocupa.

En realidad, el obstáculo más difícil de superar antes de poder cobrar peajes eficientes a los vehículos que más dañan las carreteras latinoamericanas, podría ser de carácter social o político, más que tecnológico, y estar relacionado con el poder de las federaciones de dueños de camiones, que seguramente se opondrían a las alzas de peaje. 


\section{III}

\section{¿Cómo reconocer las ineficiencias de las}

\section{estructuras de peaje en carreteras en los}

\section{procesos de privatización ferroviaria?}

\section{El sistema de bonificaciones compensato- rias a la empresa Ferrocarriles del Estado en Chile}

En el corto plazo, dentro del cual el tránsito de los camiones pesados seguirá siendo efectivamente subsidiado incluso en las carreteras concesionadas para promover una óptima distribución del tráfico entre el transporte carretero y el ferroviario, cabe contemplar compensaciones para incentivar el uso de los ferrocarriles, como sugerimos en un análisis efectuado en 1992, que consideró el tema desde el punto de vista conceptual (CEPAL, 1993).

$\mathrm{Al}$ año siguiente, en Chile se implantó un sistema de compensaciones sencillo (por t/km neta) para la empresa Ferrocarriles del Estado (pero no para las demás empresas ferroviarias del país). ${ }^{6} \mathrm{Se}$ ha anunciado que este sistema se desactivará una vez transferidas todas las operaciones ferroviarias de dicha empresa al sector privado, lo que hace pensar que el objetivo verdadero de su aplicación no habría sido mejorar la partición modal del tráfico. Ha sido criticado por varias razones; por ejemplo, después de la separación y privatización de los servicios de transporte de carga, la compensación siguió siendo pagada a Ferrocarriles del Estado, y no a la empresa operadora de los trenes de carga $(\mathrm{FdP})$, sin que hubiera garantías de que aquella la destinaría a rebajar los derechos pagados por uso de vías, o que la invertiría en mejorar la calidad de las vías ocupadas por los trenes de carga. (Una de las responsabilidades de Ferrocarriles del Estado era el mantenimiento de la infraestructura en las vías férreas principales; el mantenimiento de las vías en los ramales se ha delegado al FdP).

Sin embargo, aunque recibe críticas, el sistema de compensaciones adoptado en Chile (que se analiza más adelante desde el punto de vista conceptual) seguramente era preferible a no tener sistema alguno. En otros países ni siquiera se ha introducido un esquema

\footnotetext{
${ }^{6}$ Hubo, además, una compensación muy pequeña en términos de pasajeros/kilómetro (pax-km).
}

de ese tipo, aunque en este terreno la experiencia sueca es también interesante (Nilsson, 1993).

\section{Conveniencia de que las compensaciones a las empresas ferroviarias por los tráficos transportados se ofrezcan en la etapa de concesionamiento}

En el estudio anterior mencionado (CEPAL, 1993) analizamos las dimensiones conceptuales del problema de crear las condiciones necesarias para optimizar la partición modal. En el presente artículo, nos limitaremos a destacar la importancia de reconocer el problema al concesionarse los ferrocarriles.

Si el reconocimiento del problema es posterior, el gobierno sólo podrá resolverlo a cabalidad dictando medidas que favorezcan a una empresa del sector privado, es decir, a la propia empresa ferroviaria. Esas medidas podrían ser económicas, como una compensación por $\mathrm{t} / \mathrm{km}$ transportada por ferrocarril, o cualitativas, como la imposición de mayores restricciones sobre los pesos máximos de los camiones. En todo caso, al partido político gobernante la propuesta de tales medidas le acarrearía críticas de los partidos de la oposición, precisamente por favorecer a una empresa privada, y seguramente el sector camionero se uniría a las voces opositoras.

Por otra parte, si en las bases de la licitación se especifica la compensación que se pagará a la empresa concesionaria (o compradora) del ferrocarril, las ofertas económicas presentadas por los grupos interesados naturalmente incluirán una estimación del valor presente de los ingresos que anticipan recibir por este concepto durante el período de la concesión. En este caso, el gobierno no estaría sujeto a críticas de favoritismo.

Conviene también decidir la aplicación de compensaciones por el tráfico transportado por ferrocarril antes de concesionar las carreteras que compiten en el mismo mercado. Los beneficios generados por dichas compensaciones, que constituyen el tema principal del presente estudio, se traducen en menores costos de mantenimiento vial; evidentemente es preferible que 
ellos sean percibidos por la comunidad en general, a través del gobierno que la representa, más que por una empresa privada concesionaria de segmentos de la red vial. (Además, puede haber otros beneficios vinculados a la reducción de la congestión, los accidentes viales y la contaminación ambiental, los que serán recibidos, naturalmente, por la comunidad.)

\section{Divergencia entre lo conceptualmente preferi- ble y lo alcanzable en la realidad}

La subvención efectivamente recibida por los transportistas camioneros seguramente varía mucho de un tráfico a otro, dependiendo entre otros de los siguientes factores:

i) la incidencia de los peajes en el recorrido de los camiones dedicados al tráfico, siendo mayor la subvención al aumentarse la proporción del recorrido que se hace sobre caminos sin peaje;

ii) la categoría de las vías ocupadas, siendo mayor la subvención al subir la fracción de vías de tierra, de ripio o pavimentadas pero en mala condición;

iii) el tipo de camión, estando el subsidio inversamente relacionado con el número de ejes del vehículo, y

iv) el peso cargado en cada camión, por estar directamente relacionado el subsidio con ese peso.

Por lo tanto, conceptualmente, la compensación debería ser determinada por cada tráfico. Esto podría hacerse, por ejemplo, de acuerdo con una metodología especificada en el estudio anterior (CEPAL, 1993). Según esa metodología, antes de hacer su oferta de flete por cada tráfico licitado por el mercado, la empresa ferroviaria preguntaría al ministerio de economía o de transportes el valor de la compensación que el gobierno le pagaría, en el caso de adjudicarse el contrato de transporte.

Por otra parte, en el momento de participar en el proceso de licitación del ferrocarril y armar sus ofertas económicas correspondientes, los grupos interesados no estarán en condiciones de estimar con exactitud el volumen de cada tráfico que transportarían durante el plazo de la concesión, y tampoco podrán saber el monto que recibirían del gobierno en cada caso. Por lo tanto, desde un punto de visto práctico, en las bases se debería especificar un valor monetario que pagará el gobierno al concesionario por cada t/km transportada. Aunque este valor no equivaldría con exactitud a la compensación que correspondería a tráfico específico alguno, sí permitiría que los consorcios participantes en la licitación pudieran efectuar una estimación realista de los ingresos que obtendrían por ese concepto, e incluirlos en su oferta económica.

\section{Los beneficiarios del sistema de compensacio- nes que se propone}

En esta oportunidad, finalizaremos el presente artículo con algunos comentarios sobre los beneficiarios de un sistema de compensaciones a las empresas ferroviarias como el que se propone. En todos los casos, evidentemente, el objetivo debería ser la maximización de los beneficios percibidos por la comunidad en general.

Se pueden identificar tres situaciones diferentes, las que se analizan a continuación:

i) Cuando el ministerio de obras públicas, o su equivalente, administra por cuenta directa las redes viales, el menor volumen de tránsito de vehículos con mayor poder destructivo sobre los caminos implica una reducción correspondiente en los gastos de mantenimiento y reconstrucción de vías. La reducción de los gastos sería superior a la disminución de los ingresos al fisco por la menor recaudación tanto de impuestos sobre los combustibles como de peajes, si los hay. El gobierno podría aplicar el incremento de sus ingresos netos a otros proyectos viales o a proyectos en otras áreas, o podría rebajar los impuestos que pagan los contribuyentes. En este caso, el mecanismo de transferencia de los beneficios a la comunidad en general es bastante directo.

ii) El mecanismo es menos directo cuando las carreteras están en vías de concesionarse. En estos casos, los grupos participantes en la licitación reconocerían, o a lo menos deberían reconocer, que las compensaciones ofrecidas a las empresas ferroviarias reducirían tanto las recaudaciones por concepto de peajes como los costos de mantenimiento y reconstrucción de carreteras. Como la baja en la recaudación será absolutamente inferior a la disminución de sus costos, deberían tomar en cuenta en sus ofertas el consecuente aumento de los ingresos netos que podrá obtener el concesionario. Esto se haría en una de dos formas, dependiendo de las circunstancias. Si los valores de los peajes están definidos en las bases, cada participante subiría su oferta económica, lo que aumentaría los fondos de los que dispondría el gobierno, permitiendo financiar otros proyectos o rebajar los impuestos. Si los valores de los peajes no están definidos en las bases, cada participante reduciría el valor de los peajes que propone cobrar. En este caso, los beneficiarios serán los usuarios de la carretera que se licita, más que la comunidad en general.

iii) La situación más difícil se presenta cuando las carreteras ya han sido concesionadas, por lo cual se necesita una renegociación entre el gobierno y el concesionario. En general, la transferencia de un flujo de carga del transporte caminero al ferroviario implica un 
beneficio para el concesionario de una carretera que participa en el mismo mercado que el ferrocarril. Sin embargo, en este caso los beneficios, seguramente, tendrían que dividirse entre la comunidad, representada por su gobierno, y la empresa concesionaria.

En cualquiera de las tres situaciones descritas, bajará el costo económico del transporte, porque cada tráfico es dirigido al medio que lo pueda transportar con menor costo marginal.

\section{Pago de compensaciones a la empresa ferro- viaria sólo respecto de tráficos "nuevos"}

Según ya se ha mencionado, en 1993 el gobierno chileno decidió pagar una bonificación de 2.65 pesos (0.0066 dólares) por t/km neta a Ferrocarriles del Estado (EFE), para compensarle el subsidio efectivamente recibido por los camiones pesados, con los cuales competía.

En 1993, EFE transportaba aproximadamente 1100 millones de t/km en su Red Sur. En el gráfico 1 se hace una representación de los efectos de pagar a una empresa una compensación por $\mathrm{t} / \mathrm{km}$, basada en el caso de EFE. Se supone allí que el costo marginal del transporte de carga era de unos 16 pesos la $\mathrm{t} / \mathrm{km}$. Como EFE tenía bastante capacidad ociosa, se considera además que el costo marginal equivalía al costo medio.

Considerando que EFE negociaba contratos específicos con sus distintos clientes, cobrándoles los fletes máximos que estuviesen dispuestos a pagar, el volumen transportado por los trenes estaría dado por la ecuación $Q=2357$-78.6P, donde $Q$ representa ese volumen en millones de $\mathrm{t} / \mathrm{km}$ al año y $P$ el flete por $\mathrm{t} / \mathrm{km}$. Antes de otorgarse la bonificación, los ingresos operacionales netos por el transporte de carga habrían sido dados por el área triangular $A B C$, es decir, serían de 7689 millones de pesos.

Mediante la compensación recibida por cada t/km transportada, EFE comenzaría a recibir no solamente el flete pagado por el cliente sino también los 2.65 pesos aportados por el gobierno; luego la función que relacionaba sus ingresos con el volumen transportado habría sido la $Q=2565-78.6 P$. El volumen transportado por la empresa habría subido de 1100 a 1307 millones de $\mathrm{t} / \mathrm{km}$. Los ingresos operacionales netos de EFE se habrían elevado a 10881 millones de pesos, equivalente al área $\mathrm{FBG} .^{7} \mathrm{El}$ gobierno le habría pagado una suma correspondiente al área EFGC, es decir, 3464

\footnotetext{
${ }^{7}$ En realidad, el volumen transportado bajó en 1993, puesto que el mercado estaba disminuyendo, a raíz de factores como la baja de la producción en las minas de carbón.
}

GRAFICO 1
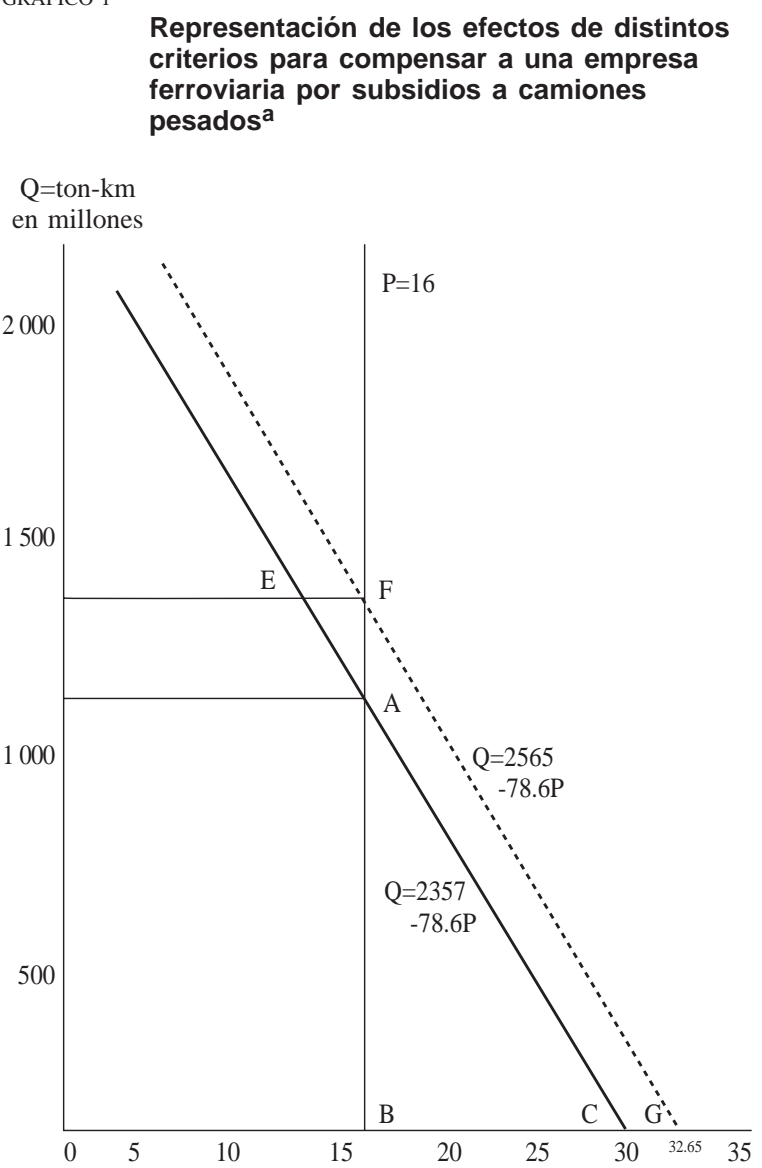

Fuente: Elaboración propia.

a Sobre la base del caso de la empresa chilena Ferrocarriles del Estado.

millones de pesos (8.66 millones de dólares). Si EFE hubiese sido una empresa privada, habría resultado políticamente inaceptable pagarle un subsidio tan alto. (Vale recordar que, en la realidad, EFE era y sigue siendo una empresa del sector público).

La compensación correspondiente al tráfico de 1100 millones de $\mathrm{t} / \mathrm{km}$ que EFE ya transportaba no habría provocado un cambio en la partición modal de las carreteras al ferrocarril; simplemente habría elevado los ingresos operacionales de EFE por transporte de carga. La empresa no habría renegociado, con fletes inferiores que antes, contratos ya firmados; pero sí habría cotizado fletes inferiores en otras licitaciones, de nuevos clientes o de los mismos, con el fin de captar tráficos. Pudo haber invertido en distintas formas de mejorar la calidad del producto ofrecido, lo que habría aumentado los volúmenes que podría transportar en años futuros; pero, en el corto plazo la suma 
respectiva, de unos 2915 millones de pesos, era meramente una transferencia del gobierno a la empresa. Por lo demás, EFE no estaba obligado a invertir sus recursos de esa manera, y sólo lo habría hecho si las rentabilidades hubiesen sido superiores a la de invertirlos en otros proyectos.

Es evidente que, en principio, el gobierno podría haber ofrecido a EFE la compensación unitaria, es decir, de 2.65 pesos la t/km sólo por los tráficos nuevos que transportase. En tal caso, el monto transferido a esta empresa habría sido de meramente 549 millones de pesos (1.37 millones de dólares), lo que habría resultado políticamente más aceptable si EFE hubiese sido una empresa privada.

Ahora bien, ¿es factible ofrecer una compensación solamente en el caso de tráficos nuevos? Hacerlo plantea ante todo el problema de que, a lo largo de los años, será cada vez más difícil distinguir los tráficos nuevos de los existentes. Considérese, por ejemplo, un contrato entre la empresa ferroviaria y una minera o industrial, con una vigencia de dos años; en sus negociaciones con el gobierno, la empresa ferroviaria podría sostener que el tráfico debería considerarse nuevo a partir del fin de la vigencia del contrato existente, y que, con posterioridad a ese momento el gobierno le debería ofrecer una compensación, siempre que se estableciese un nuevo contrato. Por lo demás, la distinción entre los tráficos existentes y los nuevos se hará aún más confusa por la reubicación de plantas generadoras de transporte, cambios en la calidad de la red de carreteras y otros factores.

También crearía dificultades el hecho de que la empresa ferroviaria percibiera por la primera unidad de tráfico nuevo transportado (en el gráfico 1, el $1100000001^{\circ} \mathrm{t} / \mathrm{km}$ ) el valor completo de la compensación, o sea, 2.65 pesos netos en el ejemplo chileno. Por otra parte, los ingresos netos que derivarían de la última unidad de tráfico existente (el $1100000000^{\circ}$ $\mathrm{t} / \mathrm{km}$ ) serían virtualmente nulos, lo que podría desembocar en que sus recursos se orientaran a atender algunos tráficos cuyo transporte por ferrocarril generara menos beneficios para el país que otros.

Se concluye que, en la práctica, no será posible, y posiblemente tampoco sea conveniente, pagar las compensaciones solamente en el caso de los tráficos nuevos.

\section{Bibliografía}

Archondo, R. (1989): EBM Case Study, Washington, D.C., Banco Mundial, septiembre.

Banco Mundial (1989): HDM-PC The Highway Design and Maintenance Standards Model, Washington, D.C., enero.

Cámara Empresarial de Larga Distancia (1998): Larga distancia, Buenos Aires, enero/febrero.

CEPAL (Comisión Económica para América Latina y el Caribe) (1987a): Sinopsis de problemas y opciones de política del transporte terrestre de carga en Chile. Estimación de los costos variables del uso de la infraestructura vial, LC/R.566/ Add.1, Santiago de Chile, 23 de julio.

(1987b): El transporte interurbano de pasajeros en Chile, LC/R.520/Rev.1, Santiago de Chile, febrero.

(1993): La metodología de la tarificación del uso de la infraestructura y la eficacia del sistema de transporte nacional, Reestructuración y privatización de los ferrocarriles de América Latina, Santiago de Chile.

(1999): El desarrollo histórico y aceptación política del concepto de la cobranza por el uso de la vialidad urbana congestionada, Santiago de Chile.

Chile, Comisión Nacional de Energía (1981): Proposición de un sistema de tarificación por uso de la infraestructura caminera, Santiago de Chile, marzo.

EFE (Ferrocarriles del Estado) (1887): $3^{a}$ Memoria de Ferrocarriles del Estado, Valparaíso, Chile, Imprenta del Universo de Guillermo Helfmann.

(varios años): Anuario estadístico, Santiago de Chile.
FLACSO (Facultad Latinoamericana de Ciencias Sociales) (1998): Transformación en el sistema vial argentino: las concesiones por peaje, Buenos Aires, febrero.

FMI (Fondo Monetario Internacional) (varios años): Estadísticas financieras internacionales, Washington, D.C.

Friedmann, J. y S. Hinojosa (sin fecha): Tarificación de la red vial interurbana; aspectos teóricos y aplicaciones, Santiago de Chile, Ministerio de Obras Públicas.

Huidobro, C. (1939): Política ferroviaria nacional, Santiago de Chile, Ministerio de Economía, Fomento y Reconstrucción.

Nilsson, J. (1993): La separación entre la propiedad de la infraestructura y la operación del transporte ferroviario: la experiencia sueca, en CEPAL. Reestructuración y privatización de los ferrocarriles. Resultados de un simposio, Santiago de Chile.

Pereira, J. (1997): Concesiones de carreteras en Brasil, trabajo presentado al Seminario Provial de las Américas, Cartagena de Indias, Colombia, septiembre.

Rolt, J. (1981): Optimum Axle Loads of Commercial Vehicles in Developing Countries, Transport and Road Research Laboratory, Informe, $\mathrm{N}^{\circ} 1002$, Crowthorne, Berkshire, Reino Unido.

Thomson, I. (1997): Red Norte: The Story of State-Owned Railways in the North of Chile, Birmingham, Reino Unido, Locomotives International.

(1998): La evolución del papel del Estado en la regulación del transporte terrestre, Revista de la CEPAL. $\mathrm{N}^{\circ} 64$, LC/G.2022-P, Santiago de Chile, CEPAL.

Transporte moderno (1996): Mayo, São Paulo, Editora TM. 